\title{
UNIQUENESS OF BOUNDED HARMONIC FUNCTIONS
}

\author{
MARVIN ORTEL AND WALTER SCHNEIDER
}

(Communicated by Irwin Kra)

\begin{abstract}
We prove the following theorem: A bounded harmonic function is identically zero if it tends to zero at a certain rate along a set of radii of positive measure. In particular, this uniqueness theorem does not require that the function in question have smooth boundary values or restricted derivatives.
\end{abstract}

\section{INTRODUCTION}

Throughout this paper, $\mathbf{R}$ denotes the set of real numbers, $\mathbf{C} \equiv \mathbf{R}^{2}$ denotes the set of complex numbers, $\mathfrak{R} z$ and $\mathfrak{I} z$ denote the real and imaginary parts of $z \in \mathbf{C}$, and $\mathscr{L}$ denotes Lebesgue outer measure on the subsets of $\mathbf{R}$.

Suppose $h$ is harmonic and bounded in the upper half plane $U \equiv\{z \in$ C: $\mathfrak{I} z>0\}$, while $E \subset \mathbf{R}, \mathscr{L}(E)>0$ and $\lim _{y \downarrow 0} h(e, y) y^{-1}=0$ for all $e \in E$. Under these assumptions, it is an open question whether $h$ is identically zero. However, if we assume in addition, that $I$ is an open interval containing $E$ and that the radial limits of $h$ are $\mathscr{L}$-equivalent to a function of smoothness class 1 on $I$, or (for a deeper result) assume that

$$
\sup _{0<y<1} \int_{I}\left|D_{1} h(x, y)\right| d \mathscr{L}(x)<\infty,
$$

it then follows that $h(z)=0$ for all $z \in U$.

The uniqueness theorem of the present paper does not require that the function in question have smooth boundary values or restricted derivatives, but does require a slightly more rapid convergence to zero on the special radii.

Theorem 1.1. Suppose $h$ is harmonic and bounded in the upper half plane $U$, $\alpha \in(0, \infty), E \subset \mathbf{R}, \mathscr{L}(E)>0$ and $\lim _{y ! 0} h(e, y) y^{-1-c}=0$ for all $e \in E$. Then $h(z)=0$ for all $z \in U$.

To prove Theorem 1.1 we must estimate $|h|$ in the channels between the special radii. For this we use the inequality of Lemma 2.3, which involves

Received by the editors August 1, 1988 .

1980 Mathematics Subject Classification (1985 Revision). Primary 31A20, 31A25.

Key' words and phrases. Bounded harmonic functions, radial limits, boundary value problems.

This research was supported by the Natural Sciences and Engineering Research Council of Canada under grant OGPIN 016. 
one of Beurling's comparison theorems for harmonic measure (Lemma 2.2). Subsequently, in Lemma 2.5, we prove that $\left|D_{1} h-i D_{2} h\right|$ tends to 0 through certain cones over the set $E$.

In $\S 3$, we prove a broader version of Theorem 1.1, which requires only that $h$ be bounded from below in cones over the set $E$, and which allows the bounds and convergence rates to vary from cone to cone. Hence, this version applies to all harmonic functions which have non-tangential limits on a boundary set of positive linear measure, and not just bounded harmonic functions. The proof uses Lemma 2.5 and Privalov's uniqueness theorem for holomorphic functions (Lemma 2.1).

\section{LEMMAS}

More notation is required to present the lemmas and the general theorem.

Depending on parameters $a, b \in \mathbf{C}, \theta \in(0, \pi / 2), r \in(0, \infty), e \in \mathbf{R}$ and $E \subset \mathbf{R}$, we define disks, horizontal segments, cones and regions formed by combining cones as follows:

$$
\begin{gathered}
D(a, r) \equiv\{z \in \mathbf{C}:|z-a|<r\}, \\
\operatorname{seg}(a, r) \equiv\{z \in \mathbf{C}: \mathfrak{I} z=\mathfrak{I} a,|z-a|<r\}, \\
c(e, \theta, r) \equiv\{(x, y) \in \mathbf{C}:|x-e|<y \tan \theta, 0<y<r\}, \\
C(E, \theta, r) \equiv \bigcup_{e \in E} c(e, \theta, r) .
\end{gathered}
$$

Hausdorff 1-dimensional (outer) measure on the subsets of $\mathbf{C}$ is denoted by $\mathscr{H}$.

We point out two properties of the regions $C(E, \theta, r)$. First, if $E$ is any subset of $\mathbf{R}$, we have

$$
C(E, \theta, r)=C(\operatorname{Clos} E, \theta, r) .
$$

Second, if $E$ is contained in an interval of length less than $2 r \tan \theta$, then $C(E, \theta, r)$ is a Jordan region with rectifiable boundary: that is, $C(E, \theta, r)$ is a bounded and connected open set, Bndry $C(E, \theta, r)$ is a Jordan curve, and $\mathscr{H}[$ Bndry $C(E, \theta, r)]<\infty$. To check the last statement, note that the lower part of Bndry $C(E, \theta, r)$ consists of $\operatorname{Clos} E \times\{0\}$ plus a sequence of similar tents erected over open segments in the complement of Clos $E$.

If $S \subset \mathbf{C}, a \in \mathbf{C}$ and $r \in(0, \infty)$, we define

$$
\mu(S, a, r) \equiv(1 / 2 r) \mathscr{H}(S \cap \operatorname{seg}(a, r)),
$$

which is the density of $S$ on $\operatorname{seg}(a, r)$. We use the same notation when $S \subset \mathbf{R}$, $a \in \mathbf{R}, \operatorname{seg}(a, r)$ is replaced by $(a-r, a+r)$ and $\mathscr{H}$ is replaced by $\mathscr{L}$ in the above definition. For typographical clarity, we define

$$
\kappa(S, a, r) \equiv(2 / \pi) \arcsin \frac{\mu(S, a, r)}{2-\mu(S, a, r)} .
$$

Note that $\mu$ and $\kappa$ vary between 0 and 1 , and that $\kappa$ is an increasing function of $\mu$. 
Suppose $J$ is a finite union of compact segments which are proper (not single points), and $r \in(0, \infty), J \subset \operatorname{seg}(0, r), z \in \operatorname{Clos} D(0, r)$ : then $\omega(z, J, D(0, r))$ denotes the value at $z$ of the harmonic measure of $J$ with respect to the region $D(0, r)-J$. Thus $\omega(\cdot, J, D(0, r))$ is the unique function which is continuous on Clos $D(0, r)$, harmonic in $D(0, r)-J$, identically 0 on Bndry $D(0, r)$ and identically 1 on $J$.

Lemma 2.1. Suppose $A \subset \mathbf{R}, \mathscr{L}(A)>0, \phi \in(0, \pi / 2)$ and $r \in(0, \infty)$. Also, suppose $\Omega$ is a connected open set which contains $C(A, \phi, r), f$ is holomorphic in $\Omega$ and $h$ is harmonic in $\Omega$. Then the following statements are true:

(1) If, for each $a \in A, \lim f(z)=0$ as $z \rightarrow(a, 0)$ through $c(a, \phi, r)$, then $f(z)=0$ for all $z \in \Omega$.

(2) If $L \in \mathbf{R}$ and $h(z) \geq L$ for all $z \in C(A, \phi, r)$, then there exists $M \in(0, \infty)$ and a compact set $B \subset \operatorname{Clos} A$ such that $\mathscr{L}(B)>0$ and

$$
|h(z)| \leq M, \quad \text { for all } z \in C(B, \phi / 2, r / 2) \text {. }
$$

Proof. (1) For $n \in\{1,2, \ldots\}$, define

$$
A_{n} \equiv\{a \in A:|f(z)| \leq 1 \text { for all } z \in c(a, \phi, 1 / n)\} .
$$

Then $A=\bigcup_{n=1}^{\infty} A_{n}$ and $\mathscr{L}(A) \leq \sum_{n=1}^{\infty} \mathscr{L}\left(A_{n}\right)$. We first select $N \in\{1,2, \ldots\}$ such that $\mathscr{L}\left(A_{N}\right)>0$, and then select a subset of $A_{N}$, of positive measure, contained in an interval of length less than $(2 / N) \tan \phi$. Replacing $A$ by this subset, and $r$ by $1 / N$, we reduce to the case that $C(A, \phi, r)$ is a Jordan region with rectifiable boundary and $f$ is bounded in $C(A, \phi, r)$. By Privalov's existence theorem ([P]), $f$ has non-tangential limits at $\mathscr{H}$-almost all points of Bndry $C(A, \phi, r)$. By hypothesis, $f(z) \rightarrow 0$ as $z \rightarrow(a, 0)$ through $c(a, \phi, r)$, for each $a \in A$ : therefore $f$ has the non-tangential limit 0 at $\mathscr{H}$-almost all $(a, 0) \in A \times\{0\}$. Since $\mathscr{H}(A \times\{0\})>0$, Privalov's uniqueness theorem ([P]) implies that $f$ is identically zero in $C(A, \phi, r)$.

(2) Again, we may assume that $C(A, \phi, r)$ is a Jordan region with rectifiable boundary. Let $f$ be holomorphic in $C(A, \phi, r)$ with $\mathfrak{R} f=h$. Since $\mathfrak{R} f$ is bounded from below, $f$ has finite non-tangential limits at $\mathscr{C}$-almost all points of Bndry $C(A, \phi, r)$ ([P]). Therefore $\sup \{|f(z)|: z \in c(a, \phi / 2, r / 2)\}<\infty$ for $\mathscr{L}$-almost all $a \in A$. For each $n \in\{1,2, \ldots\}$ define

$$
A_{n} \equiv\{a \in A:|f(z)| \leq n \text { for all } z \in c(a, \phi / 2, r / 2)\} .
$$

Choose $N \in\{1,2, \ldots\}$ such that $\mathscr{L}\left(A_{N}\right)>0$, take $B \equiv \operatorname{Clos} A_{N}$, and observe that $|h(z)| \leq|f(z)| \leq N$ for all $z \in C(B, \phi / 2, r / 2)$.

Lemma 2.2. Suppose $J$ is a finite union of proper, compact segments, $r \in(0, \infty)$ and $J \subset \operatorname{seg}(0, r)$. Then

$$
\omega(0, J, D(0, r)) \geq \kappa(J, 0, r) .
$$

Proof. Define $J^{+} \equiv\{z \in J: \mathfrak{R} z \geq 0\}$ and $\sigma \equiv(1 / r) \mathscr{H}\left(J^{+}\right)$. By symmetry, we may assume that $\sigma \geq \mu(J, 0, r)$, and hence that

$$
(2 / \pi) \arcsin \frac{\sigma}{2-\sigma} \geq \kappa(J, 0, r) .
$$


A comparison theorem of Beurling ([B]) states that

$$
\omega(0, J, D(0, r)) \geq(2 / \pi) \arcsin \frac{\sigma}{2-\sigma},
$$

and the proof is complete. Beurling's theorem also appears in [N].

We now obtain the essential inequality involving a function which is bounded and harmonic in a disk and small on a subset of the diameter.

Lemma 2.3 (Harmonic two-constant lemma). Suppose $a \in \mathbf{C} r \in(0, \infty), h$ is harmonic in $D(a, r),|h(z)| \leq 1$ for all $z \in D(a, r), m \in(0,1]$ and $S(m) \equiv$ $\{z \in D(a, r):|h(z)| \leq m\}$. Then

$$
|\tan (\pi h(a) / 4)| \leq(\tan (\pi m / 4))^{\kappa(S(m), a, r)} .
$$

Proof. It suffices to assume $a=0$ and $\kappa(S(m), 0, r)>0$. Since $h$ is harmonic, the set $S(m) \cap \operatorname{seg}(0, r)$ is a countable union of points and compact, proper segments. Thus, we may choose a set $J$, which is a finite union of compact, proper segments contained in $S(m) \cap \operatorname{seg}(0, r)$, such that $\kappa(J, 0, r)$ is as close as we wish to $\kappa(S(m), 0, r)$. Let $f$ be holomorphic in $D(0, r)$ with $\mathfrak{R} f=h$ and define, for each $z \in D(0, r)$,

$$
g(z) \equiv(1 / 2)(f(z)+\overline{f(\bar{z})}) \text { and } H(z) \equiv \tan (\pi g(z) / 4) .
$$

Then $H$ is holomorphic, $|H(x)| \leq \tan (\pi m / 4)$ for all $x \in J$ and $|H(z)| \leq 1$ for all $z \in D(0, r)$. Therefore, the two-constant theorem implies

$$
|\tan (\pi h(0) / 4)|=|H(0)| \leq(\tan (\pi m / 4))^{\omega(0, J, D(0, r))} .
$$

Since $0<\tan (\pi m / 4) \leq 1$, Lemma 2.2 gives

$$
|\tan (\pi h(0) / 4)| \leq(\tan (\pi m / 4))^{\kappa(J, 0, r)} .
$$

Since $\kappa(J, 0, r)$ is as close as we wish to $\kappa(S(m), 0, r)$, the proof is complete.

In the remaining lemmas, we show that the radial conditions on $h$ force its derivatives to tend to 0 in cones over the points at which $E$ has metric density 1 .

Lemma 2.4. Suppose $r, \beta, M \in(0, \infty), \theta \in(0, \pi / 2), \delta \in(0, r), h$ is harmonic in $c(0, \theta, r)$ and $|h(x, y)| \leq M y^{1+\beta}$ for all $(x, y) \in c(0,3 \theta / 4, \delta)$. Then

$$
\lim \left|D_{1} h(z)-i D_{2} h(z)\right|=0 \quad \text { as } z \rightarrow 0 \text { through } c(0, \theta / 2, r)
$$

Proof. Since $h$ is harmonic, there exists $C_{1} \in(0, \infty)$ such that

$$
\left|D_{1} h(z)-i D_{2} h(z)\right| \leq\left(C_{1} / \rho\right) \sup \{|h(w)|: w \in D(z, \rho)\},
$$

whenever $D(z, \rho) \subset c(0, \theta, r)$. Also, there is $C_{2} \in(0, \infty)$ such that

$$
\operatorname{dist}(z, \operatorname{Bndry} c(0,3 \theta / 4, r)) \geq C_{2} y, \quad \text { whenever } z \equiv(x, y) \in c(0, \theta / 2, \delta) \text {. }
$$

Therefore, if $z=(x, y) \in c(0, \theta / 2, \delta)$, we have

$$
|h(w)| \leq M\left(y+C_{2} y\right)^{1+\beta} \quad \text { for all } w \in D\left(z, C_{2} y\right),
$$

and

$$
\left|D_{1} h(z)-i D_{2} h(z)\right| \leq M\left(C_{1} / C_{2}\right)\left(1+C_{2}\right)^{1+\beta} y^{\beta} .
$$


Lemma 2.5. Suppose $\theta \in(0, \pi / 2), r, \alpha, L, M \in(0, \infty)$ and $E$ is a compact subset of $\mathbf{R}$. Also, assume $h$ is harmonic in $c(0, \theta, r),|h(z)| \leq L$ for all $z \in c(0, \theta, r)$ and $|h(e, y)| \leq M y^{1+\alpha}$ whenever $e \in E$ and $(e, y) \in c(0, \theta, r)$. Finally, we assume

$$
\lim _{\rho \downarrow 0} \mu(E, 0, \rho)=1
$$

and define

$$
\begin{gathered}
d(z) \equiv d(x, y) \equiv \operatorname{dist}(z, \operatorname{Bndry} c(0, \theta, r)), \\
\mu(z) \equiv \mu(x, y) \equiv \mu(E \times \mathbf{R}, z, d(z)), \\
\kappa(z) \equiv \kappa(x, y) \equiv \kappa(E \times \mathbf{R}, z, d(z)),
\end{gathered}
$$

for each $z \equiv(x, y) \in c(0, \theta, r)$. Then the following statments are valid:

(1) $\lim \mu(z)=1$ and $\lim \kappa(z)=1 \quad$ as $z \rightarrow 0$ through $c(0,3 \theta / 4, r)$;

(2) $\quad \lim \left|D_{1} h(z)-i D_{2} h(z)\right|=0 \quad$ as $z \rightarrow 0$ through $c(0, \theta / 2, r)$.

Proof. (1) If $(x, y) \in c(0,3 \theta / 4, r)$, we have

$$
-y \tan \theta<x-d(x, y)<x+d(x, y)<y \tan \theta
$$

therefore,

$$
\begin{aligned}
\mathscr{L}(E \cap(-y \tan \theta, y \tan \theta)) \leq & \mathscr{L}(E \cap(x-d(x, y), x+d(x, y))) \\
& +2 y \tan \theta-2 d(x, y) \\
= & 2 d(x, y)(\mu(x, y)-1)+2 y \tan \theta,
\end{aligned}
$$

and, upon dividing by $2 y \tan \theta$ and using the density assumption on $E$, we get

$$
\lim \inf \frac{d(x, y)}{y \tan \theta}(\mu(x, y)-1) \geq 0 \quad \text { as }(x, y) \rightarrow 0 \text { through } c(0,3 \theta / 4, r) .
$$

In the cone $c(0,3 \theta / 4, r / 2)$, the expression $d(x, y)(y \tan \theta)^{-1}$ is bounded from below by a positive constant. Therefore

$$
\liminf (\mu(x, y)-1) \geq 0 \quad \text { as }(x, y) \rightarrow 0 \text { through } c(0,3 \theta / 4, r) .
$$

Since $\mu(x, y) \leq 1$ for all $(x, y) \in c(0, \theta, r)$, the proof of the first assertion in (1) is complete and the second assertion in (1) follows immediately.

(2) Without loss of generality, we may assume $M=L=1$ and $r \in(0,1)$. If $z \equiv(x, y) \in c(0, \theta, r)$, then $|h(w)|<1$ for all $w \in D(z, d(z))$ and $|h(w)| \leq$ $y^{1+\alpha}<1$ for all $w \in(E \times(0,1)) \cap \operatorname{seg}(z, d(z))$. Thus, by Lemma 2.3, we have

$$
\left|\tan \left(\frac{\pi}{4} h(x, y)\right)\right| \leq\left(\tan \left(\frac{\pi}{4} y^{1+\kappa}\right)\right)^{\kappa(x, y)} .
$$

Choose $\beta \in(0, \alpha)$. By part (1) of the present lemma, there exists $\delta \in(0, r)$ such that $(1+\alpha) \kappa(x, y)>1+\beta$ whenever $(x, y) \in c(0,3 \theta / 4, \delta)$. Since $r \leq \tan r \leq 4 r / \pi$ for all $r \in[0, \pi / 4]$, we have

$$
|h(x, y)| \leq(4 / \pi) y^{1+\beta} \quad \text { for all }(x, y) \in c(0,3 \theta / 4, \delta) .
$$

Statement (3) now follows from Lemma 2.4. 


\section{A Generalization of Theorem 1.1}

Theorem 3.1. Suppose $\Omega$ is a connected, open subset of $\mathbf{C}, h$ is harmonic in $\Omega, E \subset \mathbf{R}$ and $\mathscr{L}(E)>0$. Also, assume that for each $e \in E$ there are corresponding numbers $\theta(e) \in(0, \pi / 2) \quad r(e), \alpha(e), M(e) \in(0, \infty)$, and $L(e) \in \mathbf{R}$ such that the following conditions hold:

(1) $c(e, \theta(e), r(e)) \subset \Omega$,

(2) $h(z) \geq L(e)$ for all $z \in c(e, \theta(e), r(e))$,

(3) $|h(e, y)| \leq M(e) y^{1+\alpha(e)}$ for all $y \in(0, r(e))$.

Then $h(z)=0$ for all $z \in \Omega$.

Proof. Consider the following special case: $E$ is compact and there exist numbers $\theta \in(0, \pi / 2), r, \alpha, L, M \in(0, \infty)$ such that $C(E, \theta, r) \subset \Omega,|h(z)| \leq L$ for all $z \in C(E, \theta, r)$ and $|h(e, y)| \leq M y^{1+\alpha}$ whenever $e \in E$ and $y \in(0, r)$. Let $A$ denote the set of all $a \in E$ for which $\lim _{\rho \downarrow 0} \mu(E, a, \rho)=1$. Since $E$ is $\mathscr{L}$-measureable, the Lebesgue metric density theorem implies $\mathscr{L}(A)=\mathscr{L}(E)>$ 0 . The special assumptions and Lemma 2.5(2) imply, for each $a \in A$,

$$
\lim \left|D_{1} h(z)-i D_{2} h(z)\right|=0 \quad \text { as } z \rightarrow(a, 0) \text { through } c(a, \theta / 2, r) .
$$

By Lemma 2.1(1), $\left|D_{1} h(z)-i D_{2} h(z)\right|=0$ for all $z \in \Omega$. Thus $h$ is constant in $\Omega$. Thus $h$ is identically zero.

We turn now to the general case. For each $n \in\{1,2, \ldots\}$, let $E_{n}$ denote the set of all $e \in E$ for which $\theta(e), r(e), \alpha(e)>1 / n, L(e)>-n$ and $M(e)<n$. Then, for some $N \in\{1,2, \ldots\}$ we have $\mathscr{L}\left(E_{N}\right)>0$ and $h(z)>-N$ for all $z \in C\left(E_{N}, 1 / N, 1 / N\right)$. By Lemma 2.1(2) we may choose a compact set $B \subset \operatorname{Clos} E_{N}$ and $L \in(0, \infty)$ so that $\mathscr{L}(B)>0$ and $|h(z)| \leq L$ whenever $z \in C(B, 1 / 2 N, 1 / 2 N)$. Since $|h(e, y)| \leq N y^{1+1 / N}$ whenever $y \in(0,1 / 2 N)$ and $e \in E_{N}$, and since $c(e, 1 / 2 N, 1 / 2 N) \subset \Omega$ whenever $e \in \operatorname{Clos} E_{N}$, it follows that $|h(b, y)| \leq N y^{1+1 / N}$ whenever $y \in(0,1 / 2 N)$ and $b \in B$. We are now reduced to the special case with $E \equiv B, \theta \equiv r \equiv 1 / 2 N, \alpha \equiv 1 / N$, $M \equiv N$ and $L$ as given above.

\section{REFERENCES}

[B] A. Beurling, Etudes sur un problème de majoration, Thèse, Uppsala, 1930.

[N] R. Nevanlinna, Analytic functions, Springer-Verlag, New York, (1970), pp. 108-111.

[P] I. I. Privalov, Randeigenschaften analytischer Funktionen, VEB Deutscher Verlag der Wissenschaften, Berlin, 1956, pp. 129-130.

Department of Mathematics, University of Hawail, Honolulu, Hawail, 96822

Department of Mathematics and Statistics, Carleton University, Ottawa, Ontario, K1S 5B6 